\title{
Test of Association in the Presence of Complex Environment
}

\author{
Muhammad Aslam (iD) and Osama H. Arif \\ Department of Statistics, Faculty of Science, King Abdulaziz University, Jeddah 21551, Saudi Arabia \\ Correspondence should be addressed to Muhammad Aslam; aslam_ravian@hotmail.com
}

Received 7 April 2020; Revised 13 May 2020; Accepted 14 May 2020; Published 28 May 2020

Academic Editor: Quanmin Zhu

Copyright (c) 2020 Muhammad Aslam and Osama H. Arif. This is an open access article distributed under the Creative Commons Attribution License, which permits unrestricted use, distribution, and reproduction in any medium, provided the original work is properly cited.

\begin{abstract}
A new test of independence under neutrosophic statistics for testing the association between two criteria of classification is presented in this paper. The necessary contingency tables for the neutrosophic population and the neutrosophic sample are presented. The test statistic of the proposed test is introduced under neutrosophic statistics. A real example from education is selected to explain the proposed test. From the real example, it is concluded that the proposed test of independence is more informative, flexible, and suitable to be applied under uncertainty as compared to the existing test under classical statistics.
\end{abstract}

\section{Introduction}

One of the important uses of the chi-square distribution is in the test of independence. In this test, the association between two categorical variables is tested using the statistic with asymptotic chi-square distribution. The test of independence is applied to test the null hypothesis that classification according to two criteria is independent versus the alternative hypothesis that classification according to two criteria is associated. The Pearson correlation is applied when the variables under study are quantities, while the test of independence is applied to see the association between qualitative variables. McHugh [1] discussed the application of the chi-square test for medical science. Singhal and Rana [2] mentioned some applications of the chi-square test. Benhamou and Melot [3] presented the graphical interpretation of the test. Lin et al. [4] introduced bootstrap for the test and applied in the biopharmaceutical industry. Kroonenberg and Verbeek [5] discussed some technical issues of the contingency table. Dutton et al. [6,7] discussed the application of the test in education. More applications of the test can be seen in [8-17].

The chi-square test of independence is designed under the assumption that all observed frequencies in the contingency table are in determined form. Nevertheless, in practice, the recorded data are not always precise and of determined form. For example, an education expert can make only the approximate estimation. In this situation, the test using the fuzzy approach is applied instead of the test under classical statistics. Runkler [18] presented the chisquare test using the fuzzy logic. Parthiban and Gajivaradhan [19] discussed the application of the fuzzy-based chisquare test in the environment. Lin et al. [20] proposed this test using membership functions. Taheri et al. [21] worked on fuzzy-based contingency tables. Alevizos et al. [22] discussed the application for education interval data. Anezakis et al. [23] applied this fuzzy approach-based test for invasive species data.

A neutrosophic logic which is the extension of the fuzzy logic is introduced by Smarandache [24]. The neutrosophic logic provides additional information that is called the measure of indeterminacy. This logic is more efficient than the fuzzy logic and interval-based analysis, see [25]. Several authors worked on neutrosophic and provided the applications, see, for example, [26-36]. Smarandache [37] introduced the neutrosophic statistics that can be applied for data having indeterminate observations using the idea of the neutrosophic logic. The neutrosophic data are expressed in neutrosophic numbers $X_{N}=X_{L}+X_{U} I_{N} ; I_{N} \in\left[I_{L}, I_{U}\right]$, where $X_{L}$ and $X_{U} I_{N}$ are determinate and indeterminate parts, respectively. Note that $I_{N} \in\left[I_{L}, I_{U}\right]$ represents the indeterminacy interval. Suppose that $I_{N} \in[0,2]$ and 
$X_{N}=2+3 I_{N}$; the neutrosophic data will be in the form of $[2,8]$, for more details, the reader may refer to [38-40] which mentioned the applications of neutrosophic numbers. Aslam et al. [41-44] introduced some statistical tests under neutrosophic statistics.

A lot of literature studies on the chi-square test of independence using the classical statistics and fuzzybased approach are available. The existing tests are unable to provide information about the measure of indeterminacy when the data are obtained from the complex system. The existing test under classical statistics can be improved using the idea of neutrosophic statistics. By exploring the literature and to the best of our knowledge, there is no work on designing the chi-square test of independence under neutrosophic statistics. In this paper, we will introduce the chi-square test of independence under indeterminacy. We will introduce the contingency tables and chi-square statistic under indeterminacy. The application of the proposed test will be given using real data from the education. We expect that the proposed test will be the best alternative of the existing tests under an uncertainty environment. The rest of the paper is organized as follows: the proposed test of independence will be discussed in Section 2. The application and comparative studies will be discussed in Sections 3 and 4 . A simulation study is given in Section 5, and some concluding remarks are given in the last section.

\section{Proposed Test of Independence}

One of the most important of the applications of the chisquare distribution is to test either the two criteria of classification are independent or not. As mentioned earlier, the existing test of independence can be applied only when the observations in the $r \times c$ contingency table are determined, where $r$ shows the rows and $c$ shows the columns of the contingency table. In this section, we propose the test of independence under the neutrosophic statistics. The classification according to two criteria of the neutrosophic population is given in Table 1 . The classification according to two criteria of the neutrosophic sample is given in Table 2. The main aim of introducing the new test of independence under the neutrosophic statistic is to test the neutrosophic null hypothesis $H_{0 N}$ that, in the neutrosophic population, two criteria are independent versus the alternative hypothesis $H_{1 N}$ that, in the neutrosophic population, two criteria are associated.

The necessary steps to evaluate the proposed test of independence under the neutrosophic statistic are explained as follows:

Step 1: state the null hypothesis $H_{0 N}$ that two criteria are independent versus the alternative hypothesis that two criteria are associated.

Step 2: specify the level of significance $\alpha$.

Step 3: compute the neutrosophic values of the expected frequency $E_{N}=\left(\left(\left(O_{N}-E_{N}\right)^{2}\right) / E_{N}\right)$.

Step 4: the proposed statistic $\chi_{N}^{2} \in\left[\chi_{L}^{2}, \chi_{U}^{2}\right]$ is given by
$\chi_{N}^{2}=\sum\left[\frac{\left(O_{N}-E_{N}\right)^{2}}{E_{N}}\right] ; \quad \chi_{N}^{2} \in\left[\chi_{L}^{2}, \chi_{U}^{2}\right], E_{N} \in\left[E_{L}, E_{U}\right]$.

The neutrosophic form of $\chi_{N}^{2} \in\left[\chi_{L}^{2}, \chi_{U}^{2}\right]$ is given by

$$
\chi_{N}^{2}=\chi_{L}^{2}+\chi_{U}^{2} I_{N} ; \quad I_{N} \in\left[I_{L}, I_{U}\right] .
$$

Note here that the proposed statistic $\chi_{N}^{2} \in\left[\chi_{L}^{2}, \chi_{U}^{2}\right]$ is the extension of the existing statistic $\chi_{L}^{2}$ used for the test of independence. The proposed test reduces to test under classical statistics when $I_{L}=0$.

Step 5: select the critical value, say $\chi_{c}^{2}$, from the chisquare table at the degree of freedom $(r-1) \times(c-1)$ at the level of significance $\alpha$.

Step 6: reject $H_{0 N}$ if $\chi_{N}^{2} \in\left[\chi_{L}^{2}, \chi_{U}^{2}\right]$ is larger than the critical value. It means $H_{0 N}$ will be rejected if the values of both $\chi_{L}^{2}$ and $\chi_{U}^{2}$ are larger than $\chi_{c}^{2}$. In some cases, it may happen that the critical value $\chi_{c}^{2}$ is between the value of $\chi_{L}^{2}$ and the value of $\chi_{U}^{2}$. In this situation, according to Smarandache [37], $H_{0 N}$ will be rejected if $\chi_{U}^{2}>\chi_{c}^{2}$; otherwise, do not reject it.

\section{Application of the Proposed Test}

In this section, we will discuss the application of the proposed test using real data from education. The education expert is interested to investigate the association between the "student's group-community" and the "department." The details about the real data can be seen in [45]. According to Alevizos et al. [22], "consider three groups of candidatestudents who are preparing to enter in a university, where each group represents a different geographical community, indicated as Student-1, Student-2, and Student-3. In order to succeed in a university, the students should choose to participate at exactly one from a choice of three directionsdepartments of the university indicated as Mathematics, Physics, and Literature." As mentioned earlier, an education expert is interested to test the null hypothesis that "student's group-community" and "department" are independent versus the alternative hypothesis that "student's groupcommunity" and "department" are associated. The data of the two criteria are taken from [22] and reported in Table 3. From Table 3, it is quite clear that the observations of "student's group-community" and "department" are presented in the neutrosophic interval. Therefore, the use of the test of independence under classical statistics may mislead the education expert. In addition, the existing test is unable to provide the probability of indeterminacy associated with the test of independence. Therefore, the education expert is interested to use the proposed test for testing the association between "student's group-community" and "department." The proposed test for the data is stated as follows:

Step 1: state the null hypothesis $H_{0 N}$ that "student's group-community" and "department" are independent versus $H_{1 N}$ that "student's group-community" and "department" are associated. 
TABLE 1: Two-way classification of the neutrosophic population.

\begin{tabular}{|c|c|c|c|c|c|c|}
\hline \multirow{2}{*}{$\begin{array}{l}2^{\text {nd }} \text { criterion of } \\
\text { classification } \\
\text { level }\end{array}$} & \multicolumn{6}{|c|}{$1^{\text {st }}$ criterion of classification level } \\
\hline & 1 & 2 & 3 & $\cdots$ & $c$ & Total \\
\hline 1 & 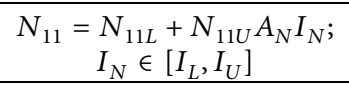 & $\begin{aligned} N_{12}= & N_{12 L}+N_{12 U} A_{N} I_{N} ; \\
& I_{N} \in\left[I_{L}, I_{U}\right]\end{aligned}$ & 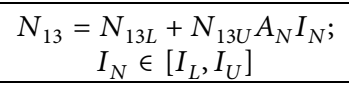 & .. & $\begin{array}{c}N_{1 c}=N_{1 c L}+N_{1 c U} A_{N} I_{N} ; \\
I_{N} \in\left[I_{L}, I_{U}\right]\end{array}$ & $N_{1}$ \\
\hline 2 & $\begin{array}{c}N_{21}=N_{21 L}+N_{21 U} A_{N} I_{N} \\
I_{N} \in\left[I_{L}, I_{U}\right]\end{array}$ & $\begin{array}{c}N_{22}=N_{22 L}+N_{222} A_{N} I_{N} ; \\
I_{N} \in\left[I_{L}, I_{U}\right]\end{array}$ & $\begin{array}{c}N_{23}=N_{23 L}+N_{23 U} A_{N} I_{N} \\
I_{N} \in\left[I_{L}, I_{U}\right]\end{array}$ & $\cdots$ & $\begin{array}{c}N_{2 c}=N_{2 c L}+N_{2 c U} A_{N} I_{N} \\
I_{N} \in\left[I_{L}, I_{U}\right]\end{array}$ & $N_{2}$ \\
\hline 3 & $\begin{array}{c}N_{31}=N_{31 L}+N_{31 U} A_{N} I_{N} \\
I_{N} \in\left[I_{L}, I_{U}\right]\end{array}$ & $\begin{aligned} N_{32}= & N_{32 L}+N_{32 U} A_{N} I_{N} ; \\
& I_{N} \in\left[I_{L}, I_{U}\right]\end{aligned}$ & $\begin{array}{c}N_{33}=N_{33 L}+N_{33 U} A_{N} I_{N} \\
I_{N} \in\left[I_{L}, I_{U}\right]\end{array}$ & & $\begin{array}{c}N_{3 c}=N_{3 c L}+N_{3 c U} A_{N} I_{N} \\
I_{N} \in\left[I_{L}, I_{U}\right]\end{array}$ & $N_{3 .}$ \\
\hline$\vdots$ & $\vdots$ & $\vdots$ & $\vdots$ & $\vdots$ & $\vdots$ & $\vdots$ \\
\hline$r$ & $\begin{array}{c}N_{r 1}=N_{r 1 L}+N_{r 1 U} A_{N} I_{N} \\
I_{N} \in\left[I_{L}, I_{U}\right]\end{array}$ & $\begin{aligned} N_{r 2}= & N_{r 2 L}+N_{r 2 U} A_{N} I_{N} \\
& I_{N} \in\left[I_{L}, I_{U}\right]\end{aligned}$ & $\begin{array}{c}N_{r 3}=N_{r 3 L}+N_{r 3 U} A_{N} I_{N} \\
I_{N} \in\left[I_{L}, I_{U}\right]\end{array}$ & & $\begin{array}{c}N_{r c}=N_{r c L}+N_{r c U} A_{N} I_{N} \\
I_{N} \in\left[I_{L}, I_{U}\right]\end{array}$ & $N_{r}$ \\
\hline Total & $N_{.1}$ & $N_{.2}$ & $N_{.3}$ & $\ldots$ & $N_{. c}$ & $N_{N}$ \\
\hline
\end{tabular}

TABle 2: Two-way classification of the neutrosophic sample.

\begin{tabular}{|c|c|c|c|c|c|c|}
\hline \multirow{2}{*}{$\begin{array}{l}2^{\text {nd }} \text { criterion of } \\
\text { classification } \\
\text { level }\end{array}$} & \multicolumn{6}{|c|}{$1^{\text {st }}$ criterion of classification level } \\
\hline & 1 & 2 & 3 & $\cdots$ & $c$ & Total \\
\hline 1 & $\begin{array}{c}n_{11}=n_{11 L}+n_{11 U} A_{N} I_{N} ; \\
I_{N} \in\left[I_{L}, I_{U}\right]\end{array}$ & $\begin{array}{c}n_{12}=n_{12 L}+n_{12 U} A_{N} I_{N} \\
I_{N} \in\left[I_{L}, I_{U}\right]\end{array}$ & $\begin{array}{c}n_{13}=n_{13 L}+n_{13 U} A_{N} I_{N} \\
I_{N} \in\left[I_{L}, I_{U}\right]\end{array}$ & & $\begin{array}{c}n_{1 c}=n_{1 c L}+n_{1 c U} A_{N} I_{N} \\
I_{N} \in\left[I_{L}, I_{U}\right]\end{array}$ & $n_{1}$ \\
\hline 2 & $\begin{array}{c}n_{21}=n_{21 L}+n_{21 U} A_{N} I_{N} ; \\
I_{N} \in\left[I_{L}, I_{U}\right]\end{array}$ & $\begin{array}{c}n_{22}=n_{22 L}+n_{22 U} A_{N} I_{N} \\
I_{N} \in\left[I_{L}, I_{U}\right]\end{array}$ & $\begin{array}{c}n_{23}=n_{23 L}+n_{23 U} A_{N} I_{N} \\
I_{N} \in\left[I_{L}, I_{U}\right]\end{array}$ & & $\begin{array}{c}n_{2 c}=n_{2 c L}+n_{2 c U} A_{N} I_{N} \\
I_{N} \in\left[I_{L}, I_{U}\right]\end{array}$ & $n_{2 .}$ \\
\hline 3 & $\begin{array}{c}n_{31}=n_{31 L}+n_{31 U} A_{N} I_{N} \\
I_{N} \in\left[I_{L}, I_{U}\right]\end{array}$ & $\begin{array}{c}n_{32}=n_{32 L}+n_{322} A_{N} I_{N} \\
I_{N} \in\left[I_{L}, I_{U}\right]\end{array}$ & $\begin{array}{c}n_{33}=n_{33 L}+n_{333} A_{N} I_{N} \\
I_{N} \in\left[I_{L}, I_{U}\right]\end{array}$ & $\cdots$ & $\begin{array}{c}n_{3 c}=n_{3 c L}+n_{3 c} A_{N} I_{N} \\
I_{N} \in\left[I_{L}, I_{U}\right]\end{array}$ & $n_{3}$ \\
\hline$\vdots$ & 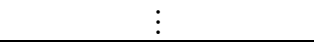 & $\begin{array}{c}\vdots \\
\end{array}$ & $\vdots$ & $\vdots$ & $\vdots$ & $\vdots$ \\
\hline$r$ & $\begin{array}{c}n_{r 1}=n_{r 1 L}+n_{r 1 U} A_{N} I_{N} \\
I_{N} \in\left[I_{L}, I_{U}\right]\end{array}$ & $\begin{array}{c}n_{r 2}=n_{r 2 L}+n_{r 2 U} A_{N} I_{N} \\
I_{N} \in\left[I_{L}, I_{U}\right]\end{array}$ & $\begin{array}{c}n_{r 3}=n_{r 3 L}+n_{r 3 U} A_{N} I_{N} \\
I_{N} \in\left[I_{L}, I_{U}\right]\end{array}$ & & $\begin{array}{c}n_{r c}=n_{r c L}+n_{r c U} A_{N} I_{N} \\
I_{N} \in\left[I_{L}, I_{U}\right]\end{array}$ & $n_{r}$ \\
\hline Total & $n_{.1}$ & $n_{.2}$ & $n_{.3}$ & $\ldots$ & $n_{. c}$ & $n_{N}$ \\
\hline
\end{tabular}

TABLe 3: Observed frequency of the real data.

\begin{tabular}{lcccc}
\hline Name & Mathematics & Physics & Literature & Total \\
\hline Student-1 & $6+10 I_{N} ; I_{N} \in[0,0.4]$ & $20+26 I_{N} ; I_{N} \in[0,0.2308]$ & $12+24 I_{N} ; I_{N} \in[0,0.5]$ & $38+60 I_{N} ; I_{N} \in[0,3.6667]$ \\
Student-2 & $15+25 I_{N} ; I_{N} \in[0,0.4]$ & $30+50 I_{N} ; I_{N} \in[0,0.4]$ & $17+17 I_{N} ; I_{N} \in[0,0]$ & $62+92 I_{N} ; I_{N} \in[0,0.3261]$ \\
Student-3 & $1+3 I_{N} ; I_{N} \in[0,0.6667]$ & $5+5 I_{N} ; I_{N} \epsilon[0,0]$ & $7+9 I_{N} ; I_{N} \epsilon[0,0.2222]$ & $13+17 I_{N} ; I_{N} \epsilon[0,0.2353]$ \\
Total & $22+38 I_{N} ; I_{N} \in[0,0.4211]$ & $55+81 I_{N} ; I_{N} \in[0,0.3209]$ & $36+50 I_{N} ; I_{N} \in[0,0.28]$ & $113+169 I_{N} ; I_{N} \in[0,0.3314]$ \\
\hline
\end{tabular}

Step 2: let $\alpha=0.05$ for this test.

Step 3: the neutrosophic values of the expected frequency $E_{N} \in\left[E_{L}, E_{U}\right]$ are shown in Table 4 .

Step 4: the calculation of the statistic $\chi_{N}^{2} \in\left[\chi_{L}^{2}, \chi_{U}^{2}\right]$ is shown as follows:

$\chi_{N}^{2}=\sum\left[\frac{\left(O_{N}-E_{N}\right)^{2}}{E_{N}}\right]=[4.66,13.43] ; \quad \chi_{N}^{2} \in[4.66,13.43]$.

The neutrosophic form of $\chi_{N}^{2} \in\left[\chi_{L}^{2}, \chi_{U}^{2}\right]$ is given by

$$
\chi_{N}^{2}=4.66+13.43 ; \quad I_{N} \in[0,0.65] \text {. }
$$

Step 5: the critical value from the chi-square table at the degree of freedom $2 \times 2$ at $\alpha=0.05$ is 9.49 .
Step 6: according to Smarandache [37], the null hypothesis is rejected if $\chi_{U}^{2}>9.49$. Therefore, the null hypothesis that "student's group-community" and "department" are independent is rejected.

\section{Comparative Study Based on Real Data}

As mentioned earlier, the proposed test of independence is the generalization of the existing test under classical statistics. For the comparison, the same level of all parameters is used for both tests. First, we compare both tests in terms of values of test statistics. Then, we will compare both tests in terms of probabilities.

The neutrosophic form of the statistic $\chi_{N}^{2} \in\left[\chi_{L}^{2}, \chi_{U}^{2}\right]$ is $\chi_{N}^{2}=4.66+13.43 ; I_{N} \in[0,0.65]$. Note here that the proposed neutrosophic form reduces to the statistic under 
TABle 4: Expected frequency of the real data.

\begin{tabular}{lccc}
\hline Name & Mathematics & Physics & Literature \\
\hline Student-1 & $7.40+13.49 I_{N} ; I_{N} \in[0,0.4514]$ & $18.49+28.76 I_{N} ; I_{N} \in[0,0.3571]$ & $12.11+17.75 I_{N} ; I_{N} \in[0,0.3177]$ \\
Student-2 & $12.07+20.68 I_{N} ; I_{N} \in[0,0.4163]$ & $30.18+44.09 I_{N} ; I_{N} \in[0,0.3155]$ & $19.75+27.22 I_{N} ; I_{N} \in[0,0.2744]$ \\
Student-3 & $2.53+3.82 I_{N} ; I_{N} \in[0,0.3377]$ & $6.33+8.15 I_{N} ; I_{N} \in[0,0.2233]$ & $4.14+5.03 I_{N} ; I_{N} \in[0,0.1770]$ \\
\hline
\end{tabular}

TABLE 5: The power of the test.

\begin{tabular}{lccc}
\hline $\mathrm{d} f$ & $\beta$ & Power of test & Power of existing test \\
\hline 1 & {$[0.04,0.01]$} & {$[0.96,0.97]$} & 0.95 \\
2 & {$[0.06,0.05]$} & {$[0.94,0.95]$} & 0.94 \\
3 & {$[0.02,0.08]$} & {$[0.91,0.94]$} & 0.9 \\
4 & {$[0.02,0.04]$} & {$[0.92,0.96]$} & 0.92 \\
5 & {$[0.09,0.06]$} & {$[0.91,0.94]$} & 0.9 \\
6 & {$[0,0.07]$} & {$[0.96,0.98]$} & 0.94 \\
7 & {$[0.04,0.04]$} & {$[0.94,0.95]$} & 0.93 \\
8 & {$[0.09,0.07]$} & {$[0.91,0.93]$} & 0.9 \\
9 & {$[0.04,0.06]$} & {$[0.9,0.94]$} & 0.9 \\
10 & {$[0.07,0.09]$} & {$[0.93,0.96]$} & 0.91 \\
20 & {$[0.04,0.01]$} & {$[0.95,0.96]$} & 0.96 \\
30 & {$[0.05,0.04]$} & {$[0.97,0.98]$} & 0.93 \\
40 & {$[0.03,0.02]$} & {$[0.95,0.96]$} & 0.95 \\
50 & {$[0.05,0.04]$} & & 0.94 \\
\hline
\end{tabular}

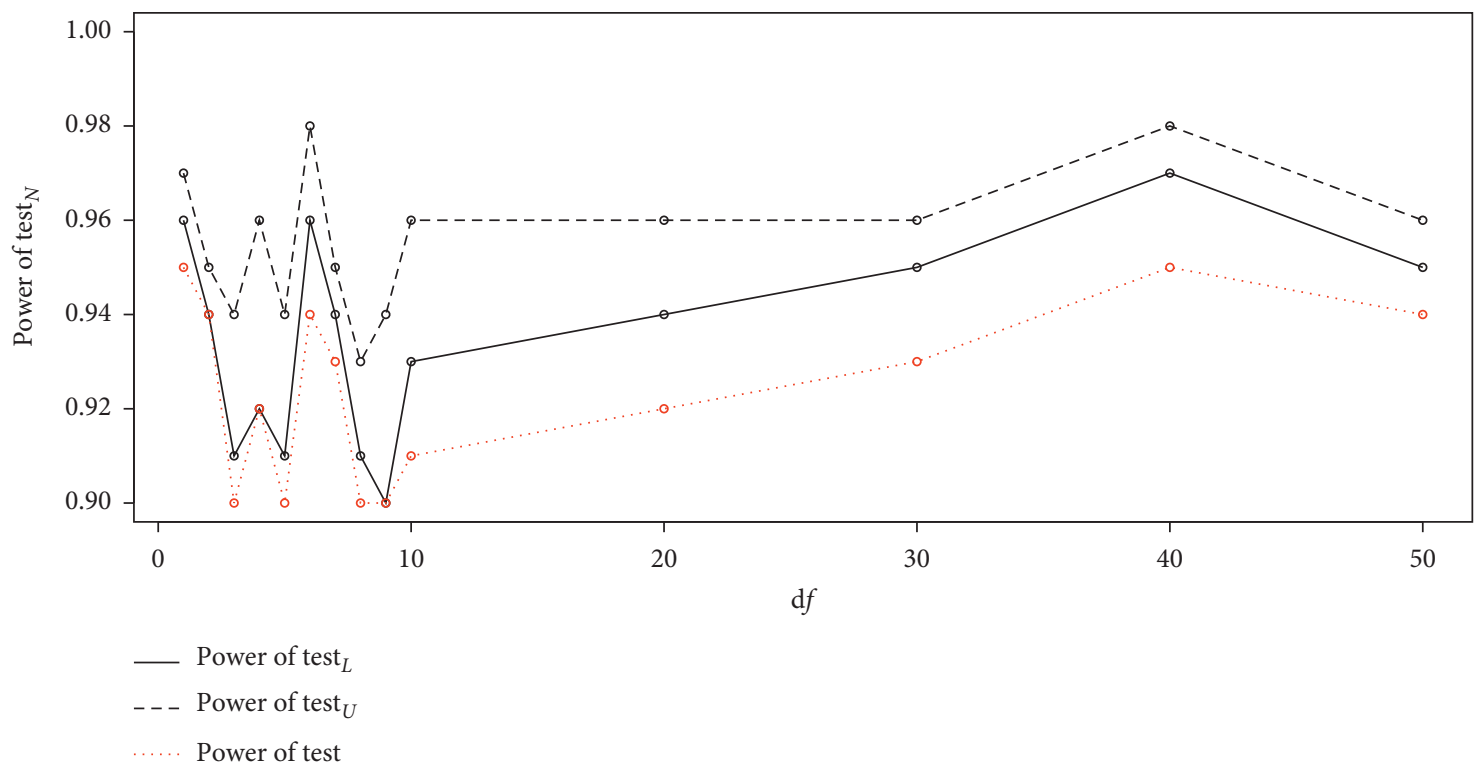

FIgURE 1: The power curves of the proposed and existing tests.

classical statistics when $I_{L}=0$. Therefore, the value of $\chi_{L}^{2}=$ 4.66 is corresponding to the statistic under classical statistics. From this result, it can be seen that the proposed test statistic values lie in the indeterminacy interval $\chi_{N}^{2} \in[4.66,13.43]$. On the contrary, the existing test under classical statistics provides only the determined value of the test statistic which is not suitable in the indeterminacy environment. From this comparison, it is concluded that the proposed test is quite affective, adequate, and effective to be applied in uncertainty as compared to the test of independence under classical statistics.
Now, we will compare both tests in terms of probabilities related to the null hypothesis. For the real data, the level of significance is $5 \%$. The existing test tells us that the chance of accepting the null hypothesis $H_{0 N}$ is 0.95 , and the probability of rejecting the null hypothesis is 0.05 . On the contrary, the proposed test tells that the chance of accepting the null hypothesis $H_{0 N}$ is 0.95 , the probability of rejecting the null hypothesis is 0.05 , and the probability of indeterminacy is 0.65 . From this comparison, it can be concluded that the proposed test provides the additional probability of indeterminacy that cannot be obtained from the existing test 
under classical statistics. Therefore, the proposed test is efficient than the existing test in providing information about the probabilities associated with the test of independence.

\section{Simulation Study}

Now, we discuss the importance of the proposed test in terms of the power of the test using simulation data. We will compare the proposed test and the existing test in terms of the power of the test. Let $\beta$ denote the type-II error which is defined as the probability of rejecting $H_{0 N}$ when $H_{0 N}$ is true. On the contrary, the power of the test $(1-\beta)$ is defined as the probability of rejecting $H_{0 N}$ when $H_{0 N}$ is false. The values of $\beta$ and $(1-\beta)$ for both tests are shown in Table 5 . The following algorithm is used to construct Table 1:

Step 1: for the $r-1 \times c-1$ contingency table, specify $\mathrm{d} f$.

Step 2: compute the values of the statistic $\chi_{N}^{2} \in\left[\chi_{L}^{2}, \chi_{U}^{2}\right]$ and compare it with $\chi_{c}^{2}$.

Step 3: compute $\beta$ by dividing the total number of accepting $H_{0 N}$ to the total number of replications, say 100.

From Table 1, it is clear that, for $\mathrm{d} f=1$ and $\mathrm{d} f=2$, the power of the existing test is between the indeterminacy of the proposed test. For $\mathrm{d} f>2$, the power of the existing test is decreasing as $\mathrm{d} f$ is increased. The power curves of both tests are shown in Figure 1. Figure 1 indicates that the proposed test has higher values of the power of the test as compared to the existing test. From this study, it can be concluded that the proposed test is better than the existing test in terms of the power of the test.

\section{Concluding Remarks}

A new test of independence under neutrosophic statistics for testing the association between two criteria of classification is presented in this paper. The necessary contingency tables for the neutrosophic population and the neutrosophic sample were presented. The test statistic of the proposed test is introduced under neutrosophic statistics. The application of the proposed test was given using the data from the education department. The comparative study shows the efficiency of the proposed test over the existing test. We recommend applying the proposed test for association testing in the presence of indeterminacy. The proposed test can be applied for big data as future research. The development of software for the proposed test is also a fruitful area of research.

\section{Data Availability}

The data are given in the paper.

\section{Conflicts of Interest}

The authors declare that they have no conflicts of interest.

\section{Acknowledgments}

The authors are thankful to Dr. Nasrullah Khan (UVAS, Pakistan) for his valuable suggestions. This work was funded by the Deanship of Scientific Research (DSR), King Abdulaziz University, Jeddah. The authors, therefore, gratefully acknowledge the DSR technical and financial support.

\section{References}

[1] M. L. McHugh, "The chi-square test of independence," Biochemia Medica, vol. 23, no. 2, pp. 143-149, 2013.

[2] R. Singhal and R. Rana, "Chi-square test and its application in hypothesis testing," Journal of the Practice of Cardiovascular Sciences, vol. 1, no. 1, p. 69, 2015.

[3] E. Benhamou and V. Melot, "Seven proofs of the pearson chisquared independence test and its graphical interpretation," 2018, https://arxiv.org/abs/1808.09171.

[4] J.-J. Lin, C.-H. Chang, and N. Pal, "A revisit to contingency table and tests of independence: bootstrap is preferred to chisquare approximations as well as Fisher's exact test," Journal of Biopharmaceutical Statistics, vol. 25, no. 3, pp. 438-458, 2015.

[5] P. M. Kroonenberg and A. Verbeek, “The tale of cochran's rule: my contingency table has so many expected values smaller than 5, what am I to do?" The American Statistician, vol. 72, no. 2, pp. 175-183, 2018.

[6] J. Dutton and M. Dutton, "Characteristics and performance of students in an online section of business statistics," Journal of Statistics Education, vol. 13, no. 3, 2005.

[7] F. Ahammed and E. Smith, "Prediction of students' performances using course analytics data: a case of water engineering course at the university of south Australia," Education Sciences, vol. 9, no. 3, p. 245, 2019.

[8] C. Kahraman, C. E. Bozdag, D. Ruan, and A. F. Özok, "Fuzzy sets approaches to statistical parametric and nonparametric tests," International Journal of Intelligent Systems, vol. 19, no. 11, pp. 1069-1087, 2004.

[9] P. Grzegorzewski, "k-sample median test for vague data," International Journal of Intelligent Systems, vol. 24, no. 5, pp. 529-539, 2009.

[10] S. N. Shahbazova, "Development of the knowledge-based learning system for distance education," International Journal of Intelligent Systems, vol. 27, no. 4, pp. 343-354, 2012.

[11] H. T. Nguyen, S. Sriboonchitta, and B. Wu, "A statistical basis for fuzzy engineering economics," International Journal of Fuzzy Systems, vol. 17, no. 1, pp. 1-11, 2015.

[12] S. R. Lipsitz, G. M. Fitzmaurice, D. Sinha, N. Hevelone, E. Giovannucci, and J. C. Hu, "Testing for independence in $J \times K$ contingency tables with complex sample survey data," Biometrics, vol. 71, no. 3, pp. 832-840, 2015.

[13] T. F. Crack, “A note on Karl Pearson's 1900 chi-squared test: two derivations of the asymptotic distribution, and uses in goodness of fit and contingency tests of independence, and a comparison with the exact sample variance chi-square result," SSRN Electronic Journal, 2018.

[14] M. Aslam, "A new failure-censored reliability test using neutrosophic statistical interval method," International Journal of Fuzzy Systems, vol. 21, no. 4, pp. 1214-1220, 2019.

[15] P. Grzegorzewski and M. Śpiewak, "The sign test and the signed-rank test for interval-valued data," International Journal of Intelligent Systems, vol. 34, no. 9, pp. 2122-2150, 2019. 
[16] P. Sulewski, "The LMS for testing independence in two-way contingency tables," Biometrical Letters, vol. 56, no. 1, pp. 17-43, 2019.

[17] J. H. Yoon, "Fuzzy mediation analysis," International Journal of Fuzzy Systems, vol. 22, no. 1, pp. 338-349, 2020.

[18] T. A. Runkler, "Fuzzy histograms and fuzzy chi-squared tests for independence," in Proceedings of the 2004 IEEE International Conference on Fuzzy Systems (IEEE Cat. No. 04CH37542), Budapest, Hungary, July 2004.

[19] S. Parthiban and P. Gajivaradhan, "A comparative study of chi-square goodness-of-fit under fuzzy environments," International Knowledge Sharing Platform, vol. 6, no. 2, 2020.

[20] P.-C. Lin, B. Wu, and J. Watada, "Goodness-of-fit test for membership functions with fuzzy data," International Journal of Innovative Computing Information and Control, vol. 8, no. 10 , pp. 7437-7450, 2012.

[21] S. M. Taheri, G. Hesamian, and R. Viertl, "Contingency tables with fuzzy information," Communications in StatisticsTheory and Methods, vol. 45, no. 20, pp. 5906-5917, 2016.

[22] P. D. Alevizos, Y. Theodorou, and M. N. Vrahatis, "Correspondence analysis with grey data: the grey eigen value problem," The Journal of Grey System, vol. 29, no. 1, pp. 92-105, 2017.

[23] V.-D. Anezakis, K. Demertzis, and L. Iliadis, "Classifying with fuzzy chi-square test: the case of invasive species," $A I P$ Conference Proceedings, vol. 1978, no. 1, 2018.

[24] F. Smarandache, Neutrosophy. Neutrosophic Probability, Set, and Logic, ProQuest Information \& Learning, vol. 105, American Research Press, Ann Arbor, MI, USA, 1998.

[25] F. Smarandache and H. E. Khalid, "Neutrosophic precalculus and neutrosophic calculus," 2015, https://arxiv.org/abs/1509/ 1509.07723 .

[26] H. Wang, F. Smarandache, Y.-Q. Zhang, and R. Sunderraman, "Interval neutrosophic sets and logic: theory and applications in computing: theory and applications in computing," 2005, https://arxiv.org/abs/cs/0505014.

[27] I. Hanafy, A. Salama, and K. Mahfouz, "Neutrosophic classical events and its probability," International Journal of Mathematics and Computer Applications Research (IJMCAR), vol. 3, no. 1, pp. 171-178, 2013.

[28] Y. Guo and A. Sengur, "NECM: neutrosophic evidential c-means clustering algorithm," Neural Computing and Applications, vol. 26, no. 3, pp. 561-571, 2015.

[29] M. Abdel-Basset, G. Manogaran, A. Gamal, and F. Smarandache, "A hybrid approach of neutrosophic sets and DEMATEL method for developing supplier selection criteria," Design Automation for Embedded Systems, vol. 22, no. 3, pp. 257-278, 2018.

[30] R. Alhabib, "Some neutrosophic probability distributions," Neutrosophic Sets and Systems, vol. 22, p. 30, 2018.

[31] S. Broumi, "Bipolar neutrosophic minimum spanning tree," SSRN Electronic Journal, 2018.

[32] X. Peng and J. Dai, "Approaches to single-valued neutrosophic MADM based on MABAC, TOPSIS and new similarity measure with score function," Neural Computing and Applications, vol. 29, no. 10, pp. 939-954, 2018.

[33] A. I. Shahin, K. M. Amin, A. A. Sharawi, and Y. Guo, "A novel enhancement technique for pathological microscopic image using neutrosophic similarity score scaling," Optik, vol. 161, pp. 84-97, 2018.

[34] M. Abdel-Basset, M. Mohamed, M. Elhoseny, L. H. Son, F. Chiclana, and A. E.-N. H. Zaied, "Cosine similarity measures of bipolar neutrosophic set for diagnosis of bipolar disorder diseases," Artificial Intelligence in Medicine, vol. 101, p. 101735, 2019.

[35] C. Jana and M. Pal, "A robust single-valued neutrosophic soft aggregation operators in multi-criteria decision making," Symmetry, vol. 11, no. 1, p. 110, 2019.

[36] N. A. Nabeeh, M. Abdel-Basset, H. A. El-Ghareeb, and A. Aboelfetouh, "Neutrosophic multi-criteria decision making approach for iot-based enterprises," IEEE Access, vol. 7, pp. 59559-59574, 2019.

[37] F. Smarandache, "Introduction to neutrosophic statistics," 2014, http://arxiv.org/abs/1406/1406.2000.

[38] J. Chen, J. Ye, and S. Du, "Scale effect and anisotropy analyzed for neutrosophic numbers of rock joint roughness coefficient based on neutrosophic statistics," Symmetry, vol. 9, no. 10, p. 208, 2017.

[39] J. Chen, J. Ye, S. Du, and R. Yong, "Expressions of rock joint roughness coefficient using neutrosophic interval statistical numbers," Symmetry, vol. 9, no. 7, p. 123, 2017.

[40] W. Jiang, J. Ye, and W. Cui, "Scale effect and anisotropic analysis of rock joint roughness coefficient neutrosophic interval statistical numbers based on neutrosophic statistics," Journal of Soft Computing in Civil Engineering, vol. 2-4, pp. 62-71, 2018.

[41] M. Aslam, "Introducing Kolmogorov-Smirnov tests under uncertainty: an application to radioactive data," ACS Omega, vol. 5, no. 1, pp. 914-917, 2020.

[42] M. Aslam, "Design of the Bartlett and Hartley tests for homogeneity of variances under indeterminacy environment," Journal of Taibah University for Science, vol. 14, no. 1, pp. 6-10, 2020.

[43] M. Aslam, O. H. Arif, and R. A. K. Sherwani, "New diagnosis test under the neutrosophic statistics: an application to diabetic patients," BioMed Research International, vol. 2020, Article ID 2086185, 7 pages, 2020.

[44] M. Aslam, "On detecting outliers in complex data using dixon's, test under neutrosophic statistics," Journal of King Saud University-Science, vol. 32, no. 3, pp. 2005-2008, 2020.

[45] Y. Theodorou, C. Drossos, and P. Alevizos, "Correspondence analysis with fuzzy data: the fuzzy eigenvalue problem," Fuzzy Sets and Systems, vol. 158, no. 7, pp. 704-721, 2007. 